translocation $\mathrm{t}(9 ; 11)(\mathrm{p} 22 ; \mathrm{q} 23)$, typically associated with the M4 or M5 French-American-British subtypes of human AML. An MLL-AF9-induced AML mouse model is widely used to study AML. Hanoun et al. showed that MLL-AF9induced AML cells disrupt bone marrow HSC niche function through alteration of the niche compartments and decrease of the expression of MSC-derived Cxcl12, Scf, Vcam1, and increased expression of Opn. ${ }^{15}$ These phenotypes in the MLL-AF9-induced AML bone marrow niche mirrored those in the Twist1-deficient bone marrow niche observed by Liu et al. ${ }^{2}$ They also found that a Twist1-deficient niche promoted progression of MLL-AF9-induced AML. By using RNAsequencing, the authors found that Jagged-2 is significantly increased in the stromal cells from the Twist1-deficient bone marrow niche and that Notch receptors are upregulated on leukemia cells. Pharmaceutical inhibition of Notch signaling partially inhibited the leukemia progression. These data indicate that AML progression is a closed loop, AML cells impair the normal bone marrow niche and alter the niche to support AML cell survival and progression. Targeting the leukemia cell - bone marrow niche loop could be an efficient strategy for leukemia therapy. Jagged-2/Notch signaling is a potential target.

\section{Relevance of understanding the hematopoietic niche in human disease}

Bone marrow failure syndromes, including aplastic anemia and myelodysplastic syndromes result from cell- and non-cell-autonomous dysregulation. Similarly, there is increasing evidence of the role of the bone marrow niche in leukemogenesis. Non-cell-autonomous dysregulation is linked to changes in the signals from innate and adaptive immune environments, and endothelial and mesenchymal lineage cells. Big data analyses based on matrix analyses of combined large data sets from single-cell RNA sequencing, flow/mass cytometry, metabolomics and proteomics mass spectrometry, as well as sophisticated microanatomical analyses are generating multiple hypotheses on the specific dissection of the interacting signal networks that connect the HSC niche cells of the bone marrow. These hypotheses require exquisite analysis and validation in mutant animal models and in therapeutic approaches when pharmacological tools to specific targets allow analyses of efficacy and safety in patients with hematologic diseases.

\section{References}

1. Gao X, Xu C, Asada N, Frenette PS. The hematopoietic stem cell niche: from embryo to adult. Development. 2018;145(2). pii: dev139691.

2. Liu X, Ma Y, Li R, et al. Niche TWIST1 is critical for maintaining normal hematopoiesis and impeding leukemia progression. Haematologica 2018;103(12):1969-1979.

3. Barnes RM, Firulli AB. A twist of insight - the role of Twist-family bHLH factors in development. Int J Dev Biol. 2009;53(7):909-924.

4. Dong CY, Liu XY, Wang N, et al. Twist-1, a novel regulator of hematopoietic stem cell self-renewal and myeloid lineage development. Stem Cells. 2014;32(12):3173-3182.

5. Arthur A, Cakouros D, Cooper L, et al. Twist-1 enhances bone marrow mesenchymal stromal cell support of hematopoiesis by modulating CXCL12 expression. Stem Cells. 2016;34(2):504-509.

6. Miraoui H, Severe N, Vaudin P, Pages JC, Marie PJ. Molecular silencing of Twist1 enhances osteogenic differentiation of murine mesenchymal stem cells: implication of FGFR2 signaling. J Cell Biochem. 2010;110(5):1147-1154

7. Zhao Z, Rahman MA, Chen ZG, Shin DM. Multiple biological functions of Twist1 in various cancers. Oncotarget. 2017;8(12):2038020393.

8. Peled A, Petit I, Kollet $O$, et al. Dependence of human stem cell engraftment and repopulation of NOD/SCID mice on CXCR4. Science. 1999;283(5403):845-848.

9. Greenbaum A, Hsu YM, Day RB, et al. CXCL12 in early mesenchymal progenitors is required for haematopoietic stem-cell maintenance. Nature. 2013;495(7440):227-230.

10. Mendez-Ferrer S, Frenette PS. Hematopoietic stem cell trafficking: regulated adhesion and attraction to bone marrow microenvironment. Ann N Y Acad Sci. 2007;1116:392-413.

11. Sharabi AB, Aldrich $M$, Sosic $D$, et al. Twist-2 controls myeloid lineage development and function. PLoS Biol. 2008;6(12):e316.

12. Wang N, Guo D, Zhao YY, et al. TWIST-1 promotes cell growth, drug resistance and progenitor clonogenic capacities in myeloid leukemia and is a novel poor prognostic factor in acute myeloid leukemia. Oncotarget. 2015;6(25):20977-20992.

13. Chen CC, You JY, Gau JP, et al. Favorable clinical outcome and unique characteristics in association with Twist1 overexpression in de novo acute myeloid leukemia. Blood Cancer J. 2015;5:e339.

14. Li X, Marcondes AM, Gooley TA, Deeg HJ. The helix-loop-helix transcription factor TWIST is dysregulated in myelodysplastic syndromes. Blood. 2010;116(13):2304-2314.

15. Hanoun M, Zhang D, Mizoguchi T, et al. Acute myelogenous leukemia-induced sympathetic neuropathy promotes malignancy in an altered hematopoietic stem cell niche. Cell Stem Cell. 2014;15(3):365375 .

\title{
Assessment of iron deficiency
}

\section{Chaim Hershko}

Professor Emeritus, Department of Hematology, Shaare Zedek Medical Center, Jerusalem, Israel

E-mail:hershkoc@netvision.net.il

doi:10.3324/haematol.2018.205575

A ccording to a study involving 187 countries, ${ }^{1}$ the global prevalence of anemia in 2010 was 33\% and it was responsible for 68 million years lived with disability. Iron deficiency was the top cause of anemia, with children below 5 years and women having the highest burden. In addition to iron deficiency, which was the most common etiology globally, other leading causes of anemia vary widely by geography, age, and sex.
Traditionally, the diagnosis of iron deficiency anemia (IDA) rests on simple measurements of serum iron, transferrin and ferritin in subjects with microcytic hypochromic anemia. However, iron deficiency and other conditions associated with anemia, such as the anemia of chronic disease and hemoglobinopathies often coexist, requiring further refinement of diagnostic strategies. The study reported by Kanuri et al. ${ }^{2}$ in this issue represents an effort to opti- 
Table 1. Diagnostic biomarkers of iron-restricted erythropoiesis.*

\begin{tabular}{lccc} 
Test & Significance & Iron deficiency anemia & Anemia of chronic disease \\
Hemoglobin g/dL & Red blood cell indices & Hb males $<13$ females $<12$ & Hb males $<13$ females $<12$ \\
MCV MCH MCHC RDW & Hypochromic microcytic & Hypo- micro- to normo \\
\hline CHr: reticulocyte $\mathrm{Hb}$ content & & Decreased & Decreased \\
HYPOr: \% retics with $\mathrm{Hb}<280 \mathrm{~g} / \mathrm{L}$ & $"$ & increased & Increased \\
\hline ZPP/H: Zinc-protoporphyrin/heme ratio & $\mathrm{RBC}$ fluorescence with Zn replacing Fe & Increased & Increased \\
\hline
\end{tabular}

\begin{tabular}{|c|c|c|c|}
\hline Serum iron & Compartment of iron in transit & Decreased & Decreased \\
\hline Transferrin & Protein carrier of iron in transit & Increased & Normal or decreased \\
\hline Transferrin saturation & Ratio of iron to transferrin & Decreased & Normal or decreased \\
\hline Ferritin & $\begin{array}{l}\text { Iron storage protein released } \\
\text { from storage sites }\end{array}$ & Decreased & Increased \\
\hline
\end{tabular}

sTfR: Soluble transferrin receptor Transferrin receptor/log ferritin

Hepcidin
Protein derived from red cell precursors Ratio of sTfR to log ferritin Liver derived peptide hormone
Increased Increased Decreased
Unchanged Unchanged

Increased

*Based on reviews by Weiss and Goodnough $2005,{ }^{19}$ Thomas et al. $2013,{ }^{20}$ Kiss $2015,{ }^{21}$ Camaschella $2015^{22}$ and Hempel and Bollard $2016 .{ }^{23}$ MCV: mean corpuscular volume; MCH mean corpuscular hemoglobin; MCHC: mean corpuscular hemoglobin concentration; RDW: red cell distribution width; Hb: hemoglobin; RBC: red blood cell; Zn: Zinc; Fe: iron.

mize diagnostic biomarkers of iron deficiency anemia employing erythrocyte zinc-protoporphyrin/heme ratio $(\mathrm{ZPP} / \mathrm{H})$ and serum hepcidin measurements.

The study by Kanuri et al. was conducted among 4 groups (90 to 100 subjects each) of subjects selected from 2227 rural community-dwelling Indian women and preschool children. It included 90 non-anemic women, 100 non-anemic children, and 100 women and 100 children with IDA. Anemia was defined as hemoglobin less than $12 \mathrm{~g} / \mathrm{dL}$ in women and less than $11 \mathrm{~g} / \mathrm{dL}$ in children. All subjects in the normal groups had serum ferritin over $30 \mathrm{ng} / \mathrm{mL}$ and all subjects in the IDA group had serum ferritin less than 12 $\mathrm{ng} / \mathrm{mL}$ and a soluble transferrin receptor (sTfR)/log ferritin index of $>2$. Subjects with low iron stores but normal hemoglobin were excluded from the study. The diagnostic performance of the biomarkers was estimated by analyzing receiver operating characteristic (ROC) curves to determine cut-off values with an optimal likelihood ratio $>10$ for IDA.

A ZPP/H ratio cut-off $>90 \mu \mathrm{mol} / \mathrm{mol}$ heme in children and $>107 \mu \mathrm{mol} / \mathrm{mol}$ heme in women was associated with a high likelihood of IDA at diagnosis (children: likelihood ratio $=20.3$, sensitivity $81 \%$ specificity $96 \%$; women: likelihood ratio $=10.873 \%$ specificity $93 \%$ sensitivity $73 \%$ ). Hepcidin cut-off values of $\leq 6.8 \mathrm{ng} / \mathrm{mL}$ in children and $\leq 4.5 \mathrm{ng} / \mathrm{mL}$ in women were associated with a high likelihood of IDA at diagnosis (children: likelihood ratio=14.3, sensitivity $86 \%$ specificity $94 \%$; women: likelihood ratio $=16.2$, sensitivity $90 \%$, specificity $94 \%$ ). The authors conclude that erythrocyte $\mathrm{ZPP} / \mathrm{H}$ ratio is a valid point-ofcare (POC) biomarker to diagnose IDA, and that the ZPP and hepcidin reference ranges and cut-off values identified in this study may guide clinicians to utilize these tests for the diagnosis of IDA in women and children.

In order to appreciate the significance of the data reported by Kanuri et al., a brief overview of our current knowledge on $\mathrm{ZPP} / \mathrm{H}$ and hepcidin measurements for evaluating iron deficiency is presented below.

\section{The zinc-protoporphyrin/heme ratio (ZPP/H)}

The use of $\mathrm{ZPP} / \mathrm{H}$ in the assessment of body iron status was reviewed in a remarkable paper by Labbe and Dewangi in 2004. ${ }^{3}$ Heme biosynthesis takes place mainly in erythroid precursor cells in the bone marrow. Iron is chelated by protoporphyrin as the final reaction in the heme pathway. This reaction is catalyzed by ferrochelatase on the mitochondrial inner membrane. Iron and zinc compete for the metal binding site of ferrochelatase and when the $\mathrm{Fe}^{2+}$ substrate is insufficient, it is substituted by $\mathrm{Zn}^{2+}$, resulting in increased $\mathrm{ZPP} / \mathrm{H}$ formation. Excess $\mathrm{ZPP} / \mathrm{H}$ formation is a reflection of iron - zinc substrate competition for ferrochelatase in iron-deficient erythropoiesis. ZPP/H is highly responsive to iron status even in borderline deficiency. Conversely, the decrease in $\mathrm{ZPP} / \mathrm{H}$ following iron supplementation in preanemic states illustrates the ability of $\mathrm{ZPP} / \mathrm{H}$ to respond to marginal changes in iron status. A major advantage of $\mathrm{ZPP} / \mathrm{H}$ measurement is the simplicity with which it can be performed, as it requires only a portable instrument, the direct reading of fluorescence without need for any reagents, and requires minimal professional training.

The ZPP/H ratio is highly specific for iron-deficient erythropoiesis. However, it does not distinguish between absolute iron deficiency and iron-deficient erythropoiesis caused by anemia of chronic disease (ACD). Thus, a positive test result of $\mathrm{ZPP} / \mathrm{H}$ should be followed by a serum ferritin determination to distinguish iron deficiency from irondeficient erythropoiesis associated with inflammation, or the toxic effect of lead exposure. Nevertheless, a ZPP/H reading within the reference range is strong evidence of adequate systemic iron supply. Indeed, as shown in a study conducted among Kenyan preschool children, when used in a screen-and-treat approach, the combination of hemoglobin concentration and whole blood $\mathrm{ZPP} / \mathrm{H}$ in a single diagnostic score can be used as a rapid and convenient testing method to rule out iron deficiency in a substantial pro- 
portion of children screened. ${ }^{4}$

Another issue of specificity is a modest increase in $\mathrm{ZPP} / \mathrm{H}$ in $\alpha$ and $\beta$ thalassemia trait. However, the combined use of red cell distribution width (RDW) or mean corpuscular volume (MCV) and $\mathrm{ZPP} / \mathrm{H}$ allows for discrimination between IDA and thalassemia trait in the vast majority of subjects. ${ }^{5,6}$

The main advantage of erythrocyte $\mathrm{ZPP} / \mathrm{H}$ measuring is the low cost, POC testing and the simplicity with which these tasks can be performed. Erythrocyte ZPP/H can be best used as a primary screening test for assessing iron status, especially in patients likely to have uncomplicated iron deficiency. In addition to its primary application, it can be useful in monitoring response to iron therapy.

\section{Hepcidin}

Hepcidin, a liver-derived peptide hormone discovered in 2001, is a key regulator of systemic iron homeostasis. . The central role of hepcidin in iron regulation has been extensively reviewed by $\mathrm{Ganz}^{8}$ and by Hentze et al., ${ }^{9}$ and the use of serum hepcidin measurements in the diagnosis of iron disorders was reviewed in 2016 by Girelli et al. ${ }^{10}$

Hepcidin functions by inhibiting the entry to the plasma of iron acquired by intestinal absorption, the recycling of iron derived from catabolism of senescent red blood cells (RBC) in macrophages, and by mobilization of iron stored in the liver. The block of iron flow is achieved by the binding of hepcidin to the iron transporter ferroportin, followed by its internalization and degradation.

Hepcidin production is increased by iron excess and by inflammation, and suppressed by both iron deficiency and increased erythropoiesis. Hepcidin production is flexible and changes within hours of introducing stimulatory or inhibitory messages such as iron administration or inflammatory stimulation. Because several opposing messages may present simultaneously, hepcidin output will depend on the relative strength of each. For example, in severe iron deficiency, hepcidin production tends to remain low, even in the presence of inflammation. Similarly, in conditions of ineffective or expanded erythropoiesis, such as in nontransfusion-dependent thalassemias, signals released by bone marrow erythroid precursors tend to override those from replete iron stores.

One such erythroid signal, erythroferrone (ERFE), has been recently identified. ${ }^{11}$ ERFE is synthesized and secreted by erythroblasts in the marrow and extramedullary sites. The production of ERFE is induced by erythropoietin and is also proportional to the total number of responsive erythroblasts. ERFE acts on hepatocytes to suppress the production of hepcidin by inhibiting hepatic BMP/SMAD signaling. By suppressing hepcidin, ERFE facilitates iron delivery during stress erythropoiesis, but also contributes to iron overload in anemias with ineffective erythropoiesis. ${ }^{12}$

The measurement of hepcidin, unlike other tests used for evaluating iron status, is a direct reflection of the mechanism controlling iron homeostasis. This unique feature of hepcidin represents a major advantage in trying to elucidate the nature of disease and its optimal management. It can be used as a guide for iron therapy. For example, it allows the prediction of favorable response to oral iron treatment among children living in countries with a high prevalence of infectious diseases, ${ }^{13}$ or the design of optimal oral iron dosing and timing by exploiting conditions that minimize iron-provoked hepcidin induction. ${ }^{14}$ It is also useful in the diagnosis of concomitant iron deficiency in patients with ACD in rheumatoid arthritis and inflammatory bowel disease, and in African children. ${ }^{15,16}$ It also allows for a rapid diagnosis of rare hereditary diseases, such as iron-refractory iron deficiency anemia (IRIDA) or ferroportin disease due to hepcidin resistant mutations. ${ }^{17,18}$

Although several assays have been developed, a gold standard is still lacking, and efforts toward harmonization are ongoing. Nevertheless, the unique advantages of hepcidin measurements can already be recognized, ranging from the use of hepcidin in diagnosing IRIDA to global health applications, such as guiding safe iron supplementation in developing countries with a high infectious disease burden.

\section{Summary}

Table 1 lists the tests currently available for evaluating iron-restricted erythropoiesis in iron deficiency and in ACD. ${ }^{19-23}$ Important considerations in the choice of diagnostic tests should be the availability, affordability, sensitivity, specificity, and minimal time required for receiving POC results. Red cell indices described in the upper 4 rows are an excellent starting point, offering knowledge regarding the duration and severity of iron deficient erythropoiesis (IDA and ACD). Because of the low specificity of red cell indices, the next set of tests should include serum iron, transferrin and ferritin. Ideally, these tests should offer a clear distinction between IDA and ACD. However, in real life, and in particular in developing countries with populations at the highest risk of anemia, IDA and ACD often coexist and the opposing directions of lab results make diagnosis difficult. This is the point where a third set of tests should be considered: ZPP/H, sTfR and hepcidin. Two of these three are the subjects of the present study by Kanuri et al. Their results show high sensitivity for IDA, but specificity could not be determined because of the design of studies pre-selecting only subjects with IDA documented by ferritin less than $12 \mathrm{ng} / \mathrm{mL}$ and an increased sTfR/log ferritin ratio.

In view of its high sensitivity and simplicity, $\mathrm{ZPP} / \mathrm{H}$ is an excellent screening procedure which may deserve inclusion in the first set of POC tests of RBC indices. In particular, it is useful in excluding iron restricted erythropoiesis whether in IDA or ACD if results are within the normal range. Both $\mathrm{sTfR}^{24}$ and hepcidin measurements are able to identify IDA in the presence of ACD. They are not inexpensive, however, and both require further efforts to turn them into universally available and validated assays. Because the measurement of hepcidin is a direct reflection of the mechanism controlling iron homeostasis, its future development into a widely available diagnostic tool may offer a major advantage in our drive to understand the nature of iron deficiency diseases and their optimal management .

\section{References}

1. Kassebaum NJ, Jasrasaria R, Naghavi M, et al. A systematic analysis of global anemia burden from 1990 to 2010. Blood. 2014;123(5):615-624

2. Kanuri G, Chichula D, Sawhne R, et al. Optimizing diagnostic biomarkers of iron deficiency anemia in community-dwelling Indian women and preschool children. Haematologica. 2018;103(12):19911996 
3. Labbé RF, Dewanji A. Iron assessment tests: transferrin receptor vis-àvis zinc protoporphyrin. Clin Biochem. 2004;37(3):165-174.

4. Teshome EM, Prentice AM, Demir AY, Andang'o PEA, Verhoef H. Diagnostic utility of zinc protoporphyrin to detect iron deficiency in Kenyan preschool children: a community-based survey. BMC Hematol. 2017:17:11

5. Tillyer ML, Tillyer CR. Zinc protoporphyrin assays in patients with alpha and beta thalassaemia trait. J Clin Pathol. 1994;47(3):205-208.

6 Harthoorn-Lasthuizen EJ, Lindemans J, Langenhuijsen MM. Combined use of erythrocyte zinc protoporphyrin and mean corpuscular volume in differentiation of thalassemia from iron deficiency anemia. Eur J Haematol. 1998;60(4):245-251.

7. Park CH, Valore EV, Waring AJ, Ganz T. Hepcidin, a urinary antimicrobial peptide synthesized in the liver. J Biol Chem. 2001;276(11):78067810.

8. Ganz T. Hepcidin and iron regulation, 10 years later. Blood. 2011;117(17):4425-4433

9. Hentze MW, Muckenthaler MU, Galy B, Camaschella C. Two to tango: regulation of mammalian iron metabolism. Cell. 2010;142(1):24-38.

10. Girelli D, Nemeth E, Swinkels DW. Hepcidin in the diagnosis of iron disorders. Blood. 2016;127(23):2809-2813.

11. Kautz L, Jung G, Valore EV, Rivella S, Nemeth E, Ganz T. Identification of erythroferrone as an erythroid regulator of iron metabolism. Nat Genet. 2014;46(7):678-684.

12. Ganz T. Erythropoietic regulators of iron metabolism. Free Radic Biol Med. 2018 Jul 5. [Epub ahead of print]

13. Prentice AM, Doherty CP, Abrams SA, et al. Hepcidin is the major predictor of erythrocyte iron incorporation in anemic African children. Blood. 2012;119(8):1922-1928.

14. Moretti D, Goede JS, Zeder C, et al. Oral iron supplements increase hepcidin and decrease iron absorption from daily or twice-daily doses in iron-depleted young women. Blood. 2015;126(17):19811989.

15. Pasricha SR, Atkinson SH, Armitage AE, et al. Expression of the iron hormone hepcidin distinguishes different types of anemia in African children. Sci Transl Med. 2014;6(235):235re3.

16. Bergamaschi G, Di Sabatino A, Albertini R, et al. Serum hepcidin in inflammatory bowel diseases: biological and clinical significance. Inflamm Bowel Dis. 2013;19(10):2166-2172

17. De Falco L, Silvestri L, Kannengiesser C, et al. Functional and clinical impact of novel TMPRSS6 variants in iron-refractory iron-deficiency anemia patients and genotype-phenotype studies. Hum Mutat. 2014;35(11):1321-1329.

18. Sham RL, Phatak PD, Nemeth E, Ganz T. Hereditary hemochromatosis due to resistance to hepcidin: high hepcidin concentrations in a family with C326S ferroportin mutation. Blood. 2009;114(2):493-494.

19. Weiss G, Goodnough LT. Anemia of chronic disease. N Engl J Med. 2005;352(10):1011-1023.

20. Thomas DW, Hinchliffe RF, Briggs C, et al. Guideline for the laboratory diagnosis of functional iron deficiency. Br J Haematol. 2013;161(5):639648.

21. Kiss JE. Laboratory and genetic assessment of iron deficiency in blood donors. Clin Lab Med. 2015;35(1):73-91.

22. Camaschella C. Iron-deficiency anemia. N Engl J Med. 2015;372 (19):1832-1843.

23. Hempel EV, Bollard ER. The evidence-based evaluation of iron deficiency anemia. Med Clin North Am. 2016;100(5):1065-1075.

24. Skikne BS, Punnonen K, Caldron PH, et al Improved differential diagnosis of anemia of chronic disease and iron deficiency anemia: a prospective multicenter evaluation of soluble transferrin receptor and the sTfR/log ferritin index. Am J Hematol. 2011;86(11):923-927.

\title{
Is DNA a better assay for residual disease in chronic myeloid leukemia?
}

\author{
Jerald Radich \\ Fred Hutchinson Cancer Research Center, Seattle, Washington, USA \\ E-mail: jradich@fhcrc.org
}

doi:10.3324/haematol.2018.205583

$\longrightarrow$ hronic myeloid leukemia (CML) is not a public health menace. Despite its rarity, it has, and continues to be, the guiding path for the concept of genetically informed medicine (here you can choose your own favorite alternative catch phrase: bench to bedside medicine, personalized medicine, precision medicine, etc.). CML was the first disease where a specific chromosomal abnormality, the Philadelphia chromosome, was identified, and the first disease where the genetic underpinnings of this chromosome abnormality were discovered (the juxtaposition of portions of the BCR gene from chromosome 22 to the tyrosine kinase domains from chromosome 9). ${ }^{1}$ This unique $B C R-A B L$ fusion gene drives the pathophysiology of the disease, and thus has led to the remarkable discovery of the tyrosine kinase inhibitors (TKIs), which have fundamentally changed the natural history of the disease. Only decades ago, the lifespan of a chronic phase CML patient was less than seven years while now these patients enjoy a survival roughly that of the normal population., ${ }^{2,3}$

Chronic myeloid leukemia has also been the model of disease monitoring using specific molecular markers. In this case, the $B C R-A B L$ chimeric RNA is used to assess disease burden, and the clinical significance of $B C R-A B L$ levels are so compelling as to drive treatment milestones based on $B C R-A B L$ levels that are codified in European and US CML guidelines. ${ }^{4 \cdot 6}$ Here, too, CML has laid the groundwork for other diseases to use so called minimal (more recently, "measurable") residual disease (MRD) to drive treatment decisions and measure clinical trial results.

In CML, $B C R-A B L$ is typically measured by testing peripheral blood RNA. RNA is used since the potential breakpoints between $B C R$ and $A B L$ cover many kilobases of DNA sequence, making an easy PCR procedure impossible. Rather, the mRNA species is predictable with only two major splicing variations, making quantitative RTPCR fairly straightforward. After considerable effort (mostly from the Adelaide group), RNA monitoring has been standardized in an International Scale, making the results comparable across more and more labs worldwide. ${ }^{6}$ The test is very sensitive, with levels of disease burden usually quantifiable to levels of four to five magnitudes from the standardized IS baseline (where a 4-log reduction of $B C R-A B L$ on the IS would equal $0.01 \%$ IS, termed MR4)

However, the RNA assay for $B C R-A B L$ is not perfect. RNA is less stable than DNA, and thus is more susceptible to transit times, temperatures, etc. ${ }^{7,8}$ This problem is ameliorated by the use of two control housekeeping genes, but these are subject to the same influences that affect the target gene, and it is perhaps a bit of a leap to 